archives-ouvertes

\title{
Single and multiple excitations in double-core-hole states of free water molecules
}

\author{
Tatiana Marchenko, S Carniato, G Goldsztejn, O Travnikova, L Journel, R
} Guillemin, I Ismail, D Koulentianos, J Martins, D Céolin, et al.

\section{To cite this version:}

Tatiana Marchenko, S Carniato, G Goldsztejn, O Travnikova, L Journel, et al.. Single and multiple excitations in double-core-hole states of free water molecules. Journal of Physics B: Atomic, Molecular and Optical Physics, IOP Publishing, 2020, 53 (22), pp.224002. 10.1088/1361-6455/abbe29 . hal03017827

\section{HAL Id: hal-03017827 \\ https://hal.sorbonne-universite.fr/hal-03017827}

Submitted on 21 Nov 2020

HAL is a multi-disciplinary open access archive for the deposit and dissemination of scientific research documents, whether they are published or not. The documents may come from teaching and research institutions in France or abroad, or from public or private research centers.
L'archive ouverte pluridisciplinaire HAL, est destinée au dépôt et à la diffusion de documents scientifiques de niveau recherche, publiés ou non, émanant des établissements d'enseignement et de recherche français ou étrangers, des laboratoires publics ou privés. 


\title{
Single and multiple excitations in double-core-hole states of free water molecules
}

\author{
T. Marchenko, ${ }^{1,2, \infty}$ S. Carniato, ${ }^{1}$ G. Goldsztejn,,${ }^{1,}$ O. Travnikova, ${ }^{1,2}$ L.

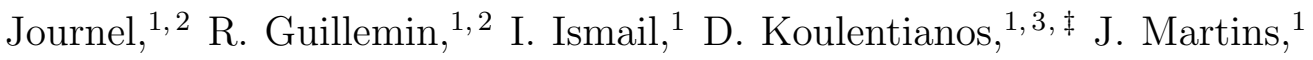 \\ D. Céolin, ${ }^{2}$ R. Püttner, ${ }^{4}$ M. N. Piancastelli, ${ }^{1,5}$ and M. Simon ${ }^{1,2}$ \\ ${ }^{1}$ Sorbonne Université, CNRS, Laboratoire de Chimie \\ Physique-Matière et Rayonnement, LCPMR, F-75005, Paris, France \\ ${ }^{2}$ Synchrotron SOLEIL, l'Orme des Merisiers, Saint-Aubin, \\ BP 48, F-91192 Gif-sur-Yvette Cedex, France \\ ${ }^{3}$ Department of Physics, University of Gothenburg, \\ Origovagen 6B, SE-41296 Gothenburg, Sweden \\ ${ }^{4}$ Fachbereich Physik, Freie Universität Berlin, \\ Arnimallee 14, D-14195 Berlin, Germany \\ ${ }^{5}$ Department of Physics and Astronomy, Uppsala University, \\ PO Box 516, SE-751 20 Uppsala, Sweden
}

(Dated: August 27, 2020)

\begin{abstract}
We present a combined experimental and theoretical study of the double-core-hole photoelectron spectrum obtained in isolated water molecules irradiated with hard x-rays above the oxygen $K^{-2}$ threshold. States of the type $\mathrm{O} K^{-2} V$ and multiply excited states are created by single-photon absorption and subsequent one-electron emission. A detailed analysis enabled by high experimental resolution reveals dissociative nuclear dynamics in the $K^{-2} V$ pre-edge states. At the binding energies above the $K^{-2}$ double-ionization potential, a complex spectral pattern is observed and attributed to highly excited states involving multiple shake-up excitation processes with the aid of state-of-the-art theoretical calculations. A strong broadening due to the nuclear motion indicates a highly dissociative nature of these multiply excited states, in agreement with the theoretical analysis.

\footnotetext{
* email: tatiana.marchenko@sorbonne-universite.fr

† Present address: Université Paris-Saclay, CNRS, Institut des Sciences Moléculaires d'Orsay, 91405, Orsay, France
} 
$\ddagger$ Present address: Chemical Sciences and Engineering Division, Argonne National Laboratory, 9700 S Cass Avenue, Lemont, IL 60439, USA 


\section{INTRODUCTION}

In the last few years, double-core-hole (DCH) spectroscopy has been well established with different experimental tools at synchrotrons (see e.g. [1-8]) and free-electron laser sources (see e.g. [9-13]).

DCH states can be formed by either simultaneous or sequential excitation and/or ejection of two core electrons [14]. The ejection of two core electrons leads to a doubly charged ion and two continuum electrons, while the simultaneous core-ionization and core-excitation process yields a singly charged species and the ejection of only one electron. The electronic states stemming from the latter mechanism are usually called DCH pre-edge states.

The most recent development has been the possibility of observing such DCH pre-edge states with good electron kinetic energy resolution by single-channel photoelectron spectroscopy and Auger measurements at synchrotron sources. Since these states correspond to the ejection of one core electron and the promotion of a second one to a virtual orbital $(V)$, they can be described within the framework of the well-known shake-up states, and considered as "super shake-ups".

The physical mechanisms leading to the formation of DCH pre-edge states can be categorized into two processes, usually described as the direct and the conjugate path. In the first case, absorption of a single photon induces dipolar ionization, leading to an ejection of a core electron to the continuum, accompanied by a monopolar excitation (monopolar "shake-up") of the second core electron. In the case of a conjugate path, absorption of a single photon results in the dipolar excitation of one core electron while the second core electron is ejected into the continuum by a monopolar "shake-off" mechanism.

The spectroscopic properties of such DCH pre-edge states were explored in several studies on isolated atoms and molecules with magnetic-bottle or HAXPES (hard x-ray photoelectron spectroscopy) spectrometers coupled with synchrotron radiation [6 8, 15, 21]. All HAXPES experiments have been conducted at the French synchrotron SOLEIL on the GALAXIES beam line, which is the first beam line in the world to allow for gas-phase spectroscopic experiments in the so-called "tender" x-ray range $(2-13 \mathrm{keV})$ with state-of-the-art photon and electron kinetic energy resolution.

A wealth of information can be derived from the DCH spectroscopy. As an example, in argon, one could identify the Rydberg series with different spin multiplicities, converging 
to the related double-ionization potential (DIP) [15]. In neon, the so-called hyper-satellite Auger spectrum, which originates from the first relaxation step of different types of DCH states, was assigned in detail [17]. In [18] $\mathrm{DCH}$ pre-edge states of $C S_{2}$ and $S F_{6}$ involving the different K-edges were presented, and the analogies and differences between the DCH photoelectron spectra and the absorption spectra of single-core-hole ( $\mathrm{SCH}$ ) states were underlined. In general, the DCH pre-edge photoelectron spectrum shows similarities to the absorption spectrum of a molecule in the presence of a core hole. However, due to a monopolar excitation in the case of formation of $K^{-2} V$ DCH pre-edge states through the direct path, population of dipole-forbidden states can occur, in particular in molecules with high symmetry, thus leading to the spectral lines, which are absent in a "standard" absorption spectrum of a molecule in the ground state. Furthermore, we have shown in several cases that continuum resonances known as shape resonances, which are present above threshold in the ionization continuum of singly ionized species, are "pushed" below threshold in DCH spectra, due to the difference in molecular potential between a single and a double core vacancy [8, 18, 21].

More recently, some papers have been published showing that not only spectroscopic but also dynamical information on DCH states can be extracted from high-resolution data. In [22] the topology of the potential energy surface and the lifetime of the DCH states of $\mathrm{CH}_{3} \mathrm{I}$ were obtained, by performing complementary measurements of resonant inelastic Xray scattering and resonant Auger spectroscopy. Furthermore, the highly repulsive nature of DCH states leading to ultrafast dissociation has been illustrated in [23, 24].

With the aim at exploiting these newly acquired experimental tools, we have turned our attention to water, the system whose dynamical properties are the most important in absolute, not only from an academic point of view, but because of the obvious links between the behavior of water molecules exposed to X-ray and important issues such as radiation damage in biological samples, "in vivo" or in water solution (see e.g. 25] and references therein).

Previous studies on DCH formation in water molecules using a magnetic-bottle spectrometer allowed identification of the oxygen $K^{-2}$ DIP and the binding energies of the first excited $K^{-2} V$ states in the DCH photoelectron spectrum [5, 7]. Direct and conjugate shake-up contributions to the $K^{-2} V$ states were identified and indications of the dissociative dynamics in these states were observed [7]. However, limited experimental resolution prevented a detailed spectral analysis. Our recent high-resolution study in gas-phase water 
using HAXPES spectrometer demonstrated the presence of nuclear dynamics, in particular the stretching of the $\mathrm{O}-\mathrm{H}$ bonds, during $\mathrm{DCH}$ formation and decay. An indication of an ultrafast proton motion was obtained through a careful analysis of the shape and width of the hyper-satellite Auger spectral lines resulting from the decay of the DCH states [26].

In this work we present a combined experimental and theoretical study of the DCH photoelectron spectrum obtained in gas-phase water molecules upon ionization with hard x-ray photons of energy well above the oxygen $K^{-2}$ threshold. At the binding energies below the DIP, the spectrum contains the previously observed pre-edge $K^{-2} V$ states. A detailed comparison of the spectral line shapes with theoretical calculations, enabled by high-resolution measurements, reveals dissociative nuclear dynamics in the pre-edge $K^{-2} V$ states. Furthermore, the experimental photoelectron spectrum exhibits broad spectral lines at the binding energies above the $K^{-2}$ DIP, which are attributed to highly excited DCH states, involving multiple shake-up excitation processes. A strong broadening due to the nuclear motion indicates a highly dissociative nature of these multiply excited states, in agreement with the theoretical analysis.

\section{METHODS}

\section{A. EXPERIMENT}

The experiment was performed at the HAXPES end station, based on a hemispherical electron analyzer installed on the GALAXIES beam line at the synchrotron SOLEIL [27, 28]. The spectrometer was set parallel to the light polarization vector. The width of the spectrometer slits was $0.8 \mathrm{~mm}$, the pass energy was set at $200 \mathrm{eV}$. The photon energy of $2.3 \mathrm{keV}$ was chosen as the lowest value available on GALAXIES. The total experimental resolution of $0.65 \mathrm{eV}$ includes the $0.6 \mathrm{eV}$ spectrometer resolution, the photon bandwidth of $0.2 \mathrm{eV}$ and the thermal Doppler broadening of $0.1 \mathrm{eV}$.

Figure 1 shows the experimental water photoelectron spectrum as a function of the binding energy. The binding-energy scale was calibrated by using the known energy positions of the $\mathrm{O} K L L$ normal Auger spectra in gas-phase water molecules [29] measured under the same experimental conditions. The photon-energy calibration was performed using the measured $\mathrm{O}$ 1s photoelectron spectrum with the known $\mathrm{O} 1 s^{-1}$ binding energy in gas-phase 


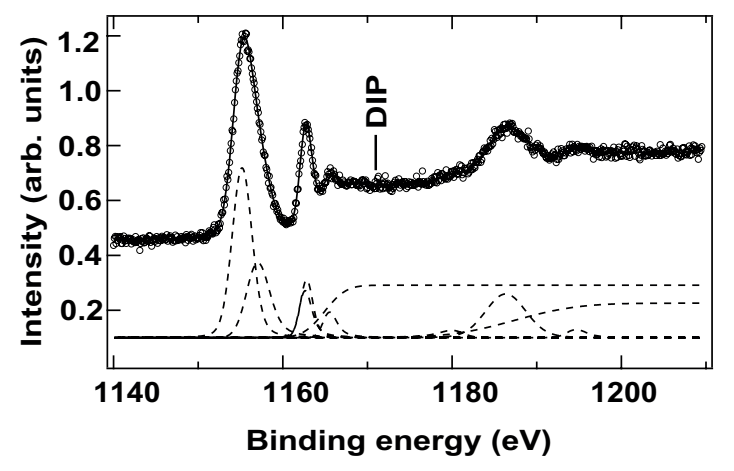

FIG. 1. Experimental photoelectron spectrum in water at $2.3 \mathrm{keV}$ photon energy (circles). Solid line through the experimental data shows the result of the fit. The components of the fit are shown in dashed lines with a vertical offset. The double ionization potential located at the binding energy of $1171 \mathrm{eV}$ is marked in the plot.

water [5]. The estimated uncertainty of the binding-energy axis of the water photoelectron spectrum resulting from the calibration procedure is on the order of $0.3 \mathrm{eV}$. The oxygen $K^{-2}$ DIP located at $1171 \mathrm{eV}[5]$ is marked in the figure. Below the DIP the spectrum contains the $K^{-2} V$ lines, previously observed and discussed in [5, 7]. The broad structure above the DIP was observed in [7], but its origin has not been discussed and so far remained unclear. High quality of the data allows for a detailed fitting, which can provide us with the information on the dynamic broadening in the excited states. In the fitting procedure we fixed the lifetime width of $0.44 \mathrm{eV} \mathrm{FWHM} \mathrm{(full} \mathrm{width} \mathrm{at} \mathrm{half} \mathrm{maximum)} \mathrm{for} \mathrm{the} \mathrm{DCH}$ state in water [30]. We also fixed the energy interval of $1.5 \mathrm{eV}$ between the two components of the first peak as known from the earlier work [5, 7]. The results of the fit are shown in figure 1. Two arctangent functions were included in the fit to describe the unresolved bound states and the continua above the DIP and above the triple ionization threshold located around $1200 \mathrm{eV}$ with two core and one valence electron missing. The energy positions and the dynamic broadening values extracted for the most prominent fit components with an estimated uncertainty of less than $0.1 \mathrm{eV}$ are summarized in table $\mathrm{I}$ and compared to the results of our calculations outlined in the next section. 


\section{B. THEORY}

A theoretical model for a description of the $K^{-2} V$ states including the direct and conjugate contributions is provided in detail in [7]. The transition energies of the $K^{-2} V$ states covering the binding energy region between $1150 \mathrm{eV}$ and $1200 \mathrm{eV}$ were calculated with GAMESS(US) quantum ab-initio package [31] using configuration interaction (CI) and density functional theory (DFT). The initial and final states were described with a post Hartree-Fock (HF) CI procedure using for oxygen an aug-cc-PCVQZ basis set augmented by additional diffuse (s,p,d) orbitals in order to better take into account Rydberg states and relaxation of the valence orbitals $(v)$ in the presence of core holes.

In our approach we use the same set of orthogonal molecular orbitals (MO) to describe both the ground state of the neutral molecule and the DCH states of the singly-ionized molecular ions. We choose a reference set consisting of MOs optimized for the $K^{-2}$ ion by HF calculations. The final-state wave functions of the molecular ions are expanded over DCH $K^{-2} V(2 h-1 e)$ configurations with two core holes and one electron excited to a vacant virtual orbital, DCH/valence-hole $K^{-2} v^{-1} V V^{\prime}(3 h-2 e)$ configurations with two core holes, one valence hole and two electrons excited to the virtual orbitals, and DCH/double-valencehole $K^{-2} v^{-1} v^{\prime-1} V V^{\prime} V^{\prime \prime}(4 h-3 e)$ configurations with two core holes, two valence holes and three electrons excited to the virtual orbitals. The initial ground state is described by a CI wave function including the main closed-shells configuration $\left(1 a_{1}\right)^{2}\left(2 a_{1}\right)^{2}\left(1 b_{2}\right)^{2}\left(3 a_{1}\right)^{2}\left(1 b_{1}\right)^{2}$, the single-valence $(v \rightarrow V)$ and double-valence $\left(v v^{\prime} \rightarrow V V^{\prime}\right)$ excited configurations as well as single-core-single-valence $\left(K v \rightarrow V V^{\prime}\right)$, single-core $(K \rightarrow V)$, and double-core $\left(K K \rightarrow V V^{\prime}\right)$ excitations.

We have computed the full $K^{-2} V$ spectrum including energies and intensities in the region below the DIP. For that, our CI-active space is restricted to single-valence and double-valence excitations and is formed by the empty $K^{-2}$ core-shell orbital, four doubly occupied valence $(v)$ MOs and sixty unoccupied virtual $(V)$ MOs.

In the region above the DIP we consider a moderate CI active space using the same set of valence occupied MOs as in the region below the DIP, but the virtual space is limited to the low-lying $4 a_{1}, 2 b_{2}, 2 b_{1}, 5 a_{1}$ unoccupied MOs, providing both the doublet states of interest contributing to the spectrum as well as the quartet ones for which DFT single-point energies were used as energies of reference. The use of a moderate active space allows for a rough 
classification by energy ordering of single and multi-excited states useful for the discussion later.

In principle, in the HF Hamiltonian of a (N)-particle system single-core and single-coresingle-valence excitations would not contribute because of the Brillouin theorem. However, since the same set of the doubly core-ionized $K^{-2}$ MOs is used for the initial state as for the $K^{-2} V$ states, single-core and single-core-single-valence excitations in the CI expansion give tiny contributions essential, respectively, for the description of the direct path in $K^{-2} V$ and $K^{-2} v^{-1} V V^{\prime}$ multi-excited states. The direct and conjugate $K^{-2} V$ cross sections are evaluated in the dipole approximation from the transition amplitudes calculated in the length gauge.

In the simulations we make an assumption that the asymmetry parameter associated to the direct shake-up is $\beta=2$ (p-wave) for the $O(1 s)$ photoelectron, and that $\beta=0$ for the conjugate shake-up (s-wave). Considering a half acceptance angle of $20^{\circ} \pm 10^{\circ}$ for the entrance lens of the analyzer, a factor $2.8 \pm 0.2$ between the direct and the conjugate shake-up pathways is taken into account [16].

For the nuclear motion, numerical gradients along the normal (anti)symmetric stretching modes are calculated within the GAMESS(US) package at a DFT level of theory, using the Becke three-parameter hybrid exchange and the Lee-Yang-Parr (B3LYP) gradient-corrected correlation functional. While below the DIP the peaks can be essentially described as an ejection of one core electron accompanied by a single discrete transition, the situation is less straightforward in the region above the DIP, where one can expect the effect of coupling between the discrete multi-excited states and the double-ionization continuum. The simulation of this region taking into account the coupling effect is out of reach due to a huge number of CI configurations.

To reproduce the region satisfactorily we adopted the following strategy. Our analysis reveals three main families of the CI states contributing to the spectrum above the DIP.

In the first case, $90 \%$ of a CI state consists of a single $(2 h-1 e)$ configuration. Since the corresponding core excitation in the presence of a core hole involves a vacant orbital located above the DIP, this state was considered as a pure continuum state. In order to calculate the continuum profile of the spectrum a static-exchange (STEX-HF) approximation technique has been adapted and combined to a Stieltjes imaging technique as described in [32].

In the second case, $90 \%$ of a CI state consists of $(3 h-2 e)$ and $(4 h-3 e)$ configurations 
corresponding to discrete multi-excitations where core excitations involve vacant orbitals located below the DIP. In this situation, similar to the one encountered for the region below the DIP, the CI state was treated as a pure discrete state.

In the third case, a CI state exhibits strong mixing between a single "continuum" state and various discrete "multiple-excitations" states. Therefore, for the sake of simplicity, these states and their associated cross sections were considered in the pool of the continuum states. The discrete multi-excitation contribution is superimposed to the simulated double continuum. Here, we assume that there is no coupling/interference between the discrete and the double continuum parts (see figure 1 in Supplementary Material for the illustration of our theoretical approach).

In the framework of our model, we consider that the line intensities derived from the direct path are only due to single-core $K \rightarrow V$ and single-core-single-valence $K v \rightarrow V V^{\prime}$ transitions implemented in the initial ground state CI wave-function. However, while such core-valence excited configurations play a minor role in the neutral state to produce multi-electronic excited states above the DIP, the same configurations can in principle be also formed from double-core excited configurations (preserving symmetry conditions) contributing to the neutral ground state.

As an example, the $K^{-2} v^{-1} V V^{\prime}$ final configuration can be reached from a direct path, i.e. by dipolar ejection to the continuum of one $O(1 s)$ electron from the initial $K^{-1} v^{-1} V V^{\prime}$ configuration. Alternatively, it can be obtained by a single valence ionization $v \rightarrow v^{-1}$ following x-ray photon absorption from the $K^{-2} V V^{\prime}$ electronic configuration contributing to the neutral CI description. This valence ionization channel could thus interfere with the core-ionization direct path, possibly impacting the cross sections in the region above the DIP (see discussion in [33] and references therein). While we explicitly treat the double-core excitations in the CI neutral state, the interference effects were not considered, since those are beyond the purpose of the present study. 

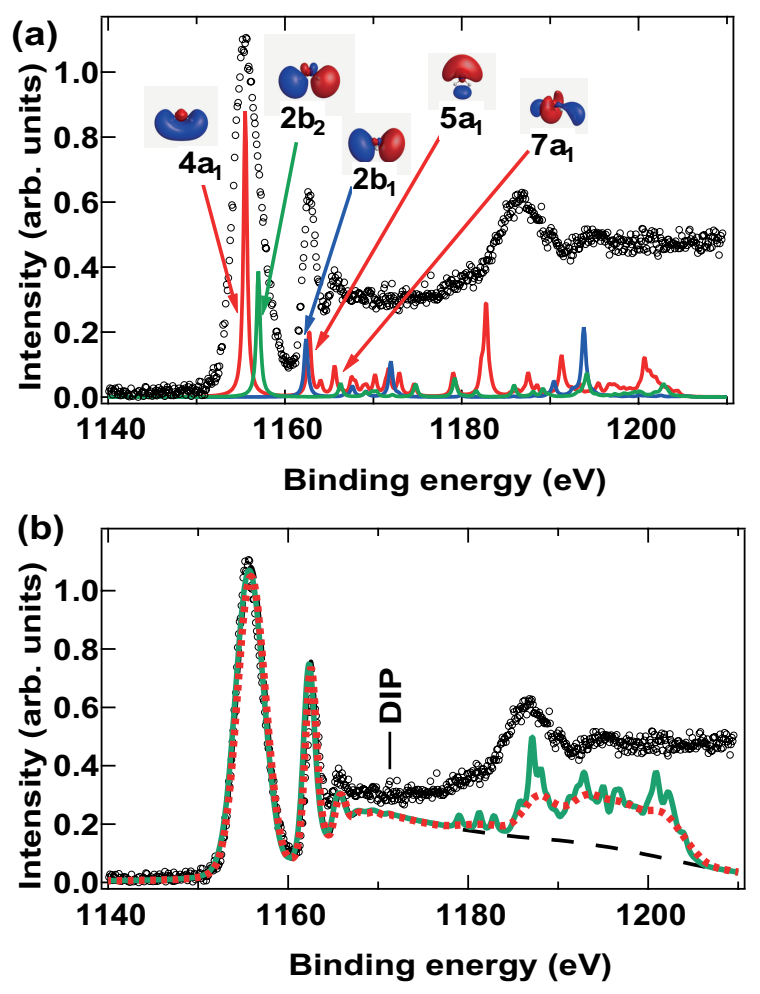

FIG. 2. (a) Comparison of an experimental photoelectron spectrum of water measured at 2.3 $\mathrm{keV}$ (circles) with the CI calculated spectrum consisting of discrete $K^{-2} V$ lines broadened by the oxygen $K^{-2}$ lifetime width. Contributions of states with different symmetries are shown with solid lines of different colors: $a_{1}$ red, $b_{2}$ green, $b_{1}$ blue. Assignment of the molecular orbitals involved in the core excitation is shown for the most prominent lines below the DIP. (b) Comparison of the experimental photoelectron spectrum (circles) to the calculations treating the states above the DIP as pure continuum states (dashed black) and as a mixture of continuum and discrete states broadened by a Gaussian of $0.4 \mathrm{eV} \mathrm{FWHM} \mathrm{(solid} \mathrm{green)} \mathrm{or} 4 \mathrm{eV} \mathrm{FWHM} \mathrm{(dotted} \mathrm{red).}$

\section{RESULTS AND DISCUSSION}

\section{A. Region below the DIP}

Figure 2(a) shows the assignment of the MOs involved in the $\mathrm{O} 1 s \rightarrow V$ core excitations contributing to the region below the DIP. The CI calculated discrete $K^{-2} V$ lines are broadened by a Lorentzian with the oxygen $K^{-2}$ lifetime width of $0.44 \mathrm{eV}$ FWHM [30]. For the states of $a_{1}$ symmetry, the contributions of the direct and the conjugate paths are summed up, whereas for the states of other symmetries, $b_{1}$ and $b_{2}$, only the conjugate pathway re- 
mains possible [7]. The binding energies of the first four $K^{-2} V$ excited states calculated at CI and DFT levels of theory are summarized in table I. A constant offset is applied to the calculated binding energies to align the $1 a_{1}^{-2} 4 a_{1}$ peak with the maximum of the first experimental peak.

In order to clarify the possible role of the nuclear motion during the $\mathrm{DCH}$ formation, the widths of Franck-Condon (FC) factors for the first four $K^{-2} V$ states were obtained from calculation of the gradients of the potential energy surfaces along the various normal modes at the neutral equilibrium geometry. At the equilibrium geometry of the initial electronic ground state, the largest gradient is for the symmetric stretching mode, while the component along the bending mode is much weaker and vanishes for the antisymmetric stretching mode.

For the main broad peak, formed by the $1 a_{1}^{-2} 4 a_{1}$ and $1 a_{1}^{-2} 2 b_{2}$ states, the FC values of $2.4 \mathrm{eV}$ and $2.8 \mathrm{eV}$, respectively, obtained from the fit of the experimental data, are in good agreement with the calculations (see table II). The reported values are slightly larger than the theoretical FC broadening of $2.2 \mathrm{eV}$ FWHM provided for the DCH states in water in our recent paper [26]. The large gradients along the symmetric stretching mode, shown in table If can be explained by the strong antibonding character of the corresponding MOs.

Gradient calculation must be considered with caution since the dynamics in the FC zone is determined by the potential energy surface corresponding to the symmetric stretching mode. However, outside the FC zone, i.e far from the neutral equilibrium geometry, a switch to the the antisymmetric stretching mode can occur leading to $\mathrm{OH}-\mathrm{H}$ bond dissociation [8].

The experimental width of the second most intense peak in the spectrum is considerably reduced compared to the main peak. The FC values of $1.2 \mathrm{eV}$ and $1.4 \mathrm{eV}$ obtained from the fit of the contributing $1 a_{1}^{-2} 2 b_{1}$ and $1 a_{1}^{-2} 5 a_{1}$ states are in good agreement with the theoretical results. The corresponding values of the gradients along the symmetric stretching mode are roughly two times lower compared to the case of the $1 a_{1}^{-2} 4 a_{1}$ and $1 a_{1}^{-2} 2 b_{2}$ states (see table II). This is due to the fact that $2 b_{1}$ is a non-bonding MO and $5 a_{1}$ is much more diffuse than $4 a_{1}$ or $2 b_{2}$. It is interesting to note that full geometry optimization leads also to final $\mathrm{OH}+\mathrm{H}^{+}$ fragments in both cases indicating that the $K^{-2} V$ states are metastable at the equilibrium geometry (the gradient along the antisymmetric mode is equal to zero) and should present specific dynamics involving the antisymmetric stretching mode outside the FC zone.

The question of nuclear dynamics in core-ionized water molecules has a relatively long history. It is well known that upon resonant core excitation below the $\mathrm{O}(1 \mathrm{~s})$ single-ionization 
threshold the water molecule undergoes ultrafast dissociation, meaning that there is a competition between resonant Auger decay from the intact molecule and $\mathrm{O}-\mathrm{H}$ bond rupture during the core-hole lifetime of around $5 \mathrm{fs}$, and therefore, in the resonant Auger spectra one can identify spectral features related to the decay of the intact molecular ion as well as of the $\mathrm{OH}$ fragment [34].

In the case of DCH state formation with a much shorter lifetime of $1.5 \mathrm{fs}$, no complete dissociation can be expected. However, since the nuclear motion starts within the DCH lifetime, one can expect its fingerprint in the DCH spectra. As we have recently shown, the nuclear dynamics has to be taken into account for an accurate description of the hyper-satellite Auger spectra resulting from the decay of the DCH states in water [26]. In particular, we

have shown there that the $1 a_{1}^{-2} 4 a_{1}$ state presents a large dynamical effect, due mainly to the anti-bonding character of the $4 a_{1} \mathrm{MO}$.

The previous attempts to reveal the presence of nuclear motion in the DCH photoelectron spectra of water were limited by the poor experimental resolution exceeding the FC broadening of the dissociative state [5, 7]. In contrast, our high experimental resolution allows for an unambiguous extraction of the FC broadening of the first four $K^{-2} V$ states and therefore, reveals a clear manifestation of nuclear dynamics in these DCH states.

One can expect that in heavy water the dissociative dynamics of the molecule will be reduced, similar to the previous observation in singly core-excited water [34]. Provided the practically identical gradients of the PES in $\mathrm{H}_{2} \mathrm{O}$ and $\mathrm{D}_{2} \mathrm{O}$ in a core-excited state in the equilibrium geometry, the dynamic FC broadening is determined by the width of the vibrational wavefunction in the ground state, which in general depends on the masses of the atomic constituents of the molecule and on the vibrational frequency along a given mode [35]. For a symmetric stretching mode in the harmonic approximation and with a static oxygen atom, a replacement of the hydrogen atoms by two times heavier isotopes, will lead to a stronger localization of the vibrational wavefunction around the equilibrium interatomic distance, providing a reduction of the $\mathrm{FC}$ widths by a factor of $\sqrt{2}$.

\section{B. Region above the DIP}

The experimental spectrum above the DIP contains an intense broad peak at the binding energy of $1186.3 \mathrm{eV}$ and two smaller contributions located around $1180 \mathrm{eV}$ and 1194 
TABLE I. Experimental and theoretical binding energies calculated at CI and single-point DFT/B3LYP level, Franck-Condon widths and calculated gradients of the potential energy surfaces in the FC zone for several characteristic DCH states in water located below and above the DIP. Uncertainties in experimental energy and FC width are given for the last significant digit in parentheses.

\begin{tabular}{|c|c|c|c|c|c|c|}
\hline Configuration & Energy $_{e x p}, \mathrm{eV}$ & Energy CI, eV & Energy DFT, eV & $F C_{e x p}, \mathrm{eV}$ & $F C_{t h}, \mathrm{eV}$ & Grad., eV/Å \\
\hline \multicolumn{7}{|l|}{ Below the DIP } \\
\hline $1 a_{1}^{-2} 4 a_{1}$ & $1155.2(3)$ & 1155.5 & 1155.5 & $2.4(1)$ & 2.6 & 16.8 \\
\hline $1 a_{1}^{-2} 2 b_{2}$ & $1156.7(3)$ & 1157.0 & 1156.6 & $2.8(1)$ & 2.8 & 18.2 \\
\hline $1 a_{1}^{-2} 2 b_{1}$ & $1162.5(3)$ & 1162.4 & 1162.3 & $1.2(1)$ & 1.4 & 8.9 \\
\hline $1 a_{1}^{-2} 5 a_{1}$ & $1162.8(3)$ & 1162.7 & 1162.6 & $1.4(1)$ & 1.5 & 9.0 \\
\hline \multicolumn{7}{|l|}{ Above the DIP } \\
\hline \multicolumn{7}{|l|}{ Group 1} \\
\hline & 1180 & & & & & \\
\hline $1 a_{1}^{-2} 1 b_{2}^{-1} 4 a_{1} 2 b_{2}$ & & & 1179.17 & & 6.8 & 43.5 \\
\hline $1 a_{1}^{-2} 3 a_{1}^{-1} 4 a_{1}^{2}$ & & & 1179.45 & & 5.7 & 36.9 \\
\hline \multicolumn{7}{|l|}{ Group 2} \\
\hline & 1186.3 & & & & & \\
\hline $1 a_{1}^{-2} 1 b_{1}^{-1} 4 a_{1} 2 b_{1}$ & & & 1183.49 & & 4.4 & 28.7 \\
\hline $1 a_{1}^{-2} 3 a_{1}^{-1} 4 a_{1} 5 a_{1}$ & & & 1184.56 & & 4.6 & 29.8 \\
\hline $1 a_{1}^{-2} 1 b_{2}^{-1} 2 b_{2} 5 a_{1}$ & & & 1187.56 & & 5.5 & 35.7 \\
\hline \multicolumn{7}{|l|}{ Group 3} \\
\hline & 1194 & & & & & \\
\hline $1 a_{1}^{-2} 1 b_{1}^{-1} 5 a_{1} 2 b_{1}$ & & & 1192.45 & & 3.2 & 20.6 \\
\hline $1 a_{1}^{-2} 3 a_{1}^{-1} 2 b_{1} 5 a_{1}$ & & & 1193.60 & & 3.3 & 21.4 \\
\hline
\end{tabular}

$\mathrm{eV}$. These features are not at all reproduced by theory within a discrete basis set used in figure 2(a). On the other hand, the simulations considering all the states above the DIP as a pure continuum, yield a flat dashed curve in figure 2(b) that also fails to reproduce the experimentally observed structures.

Therefore, it is important to take into account the nature (discrete/continuum) of the dif- 
ferent states contributing to this energy region, formed by multiple shake-up configurations, where one core electron is ejected into the continuum, the second core electron is excited to one of the vacant MOs $\left(4 a_{1}, 2 b_{2}, 2 b_{1}, 5 a_{1}, 6 a_{1}\right)$ and a third (valence-shell) electron is shaken from an occupied MO $\left(2 a_{1}, 1 b_{2}, 3 a_{1}, 1 b_{1}\right)$ to a vacant MO.

The binding energies, gradients and corresponding FC widths, calculated at single-point DFT/B3LYP level of theory, are shown in table If. For the open-shell final states the spin quartet configurations were considered (see table 1 in Supplementary Material for detailed theoretical results).

Several selected multiple shake-up states listed in table I are split in three groups according to their energies. The first group contains the configurations with one core-shell $1 a_{1}$ electron ejected to the continuum, whereas the other $1 a_{1}$ electron and a valence electron are shaken to the lowest vacant $4 a_{1}$ or $2 b_{2}$ MOs. These lowest unoccupied MOs are involved in the $K^{-2} V$ states forming the first peak below the DIP at the lowest binding energy around $1155.5 \mathrm{eV}$. The binding energies of the states in the first group above the DIP are close to $1180 \mathrm{eV}$, where the experimental data contains a small peak.

The second group contains the states with binding energies close to the most intense broad peak observed above the DIP at $1186.3 \mathrm{eV}$. The states in this group have one electron excited to one of the lowest vacant $\operatorname{MOs} 4 a_{1}$ or $2 b_{2}$, and another electron promoted to an outer $5 a_{1}$ or $2 b_{1}$ MOs. For instance, the peak with the DFT binding energy of $1184.56 \mathrm{eV}$ corresponds to the final configuration $1 a_{1}^{-2} 3 a_{1}^{-1} 4 a_{1} 5 a_{1}$, where one core-shell $1 a_{1}$ electron is ejected to the continuum, the other $1 a_{1}$ electron is promoted to the vacant MO $4 a_{1}$, and another electron is shaken from a deep-valence $3 a_{1}$ to the outer $5 a_{1}$ MO. The outer MOs $5 a_{1}$ and $2 b_{1}$ are involved in the $K^{-2} V$ states forming the second peak below the DIP at the binding energy around $1162.5 \mathrm{eV}$.

The third group contains the configurations where both the core-shell and a valence electrons are excited to the outer $5 a_{1}$ or $2 b_{1}$ MOs. The binding energies of these states are the highest of the three groups and are close to $1194 \mathrm{eV}$, where we observe a small peak in the experimental data.

The gradient analysis for these single-configuration states indicates a strong repulsive behaviour along the symmetric stretching mode. This is not very surprising since in these cases the core excitation into an antibonding $\mathrm{MO}\left(4 a_{1}, 2 b_{2}, 2 b_{1}\right.$ or $\left.5 a_{1}\right)$ is accompanied by an additional valence excitation from a bonding MO $\left(1 b_{1}, 3 a_{1}, 1 b_{2}\right)$ to an antibonding MO, 
thus reducing the strength of the $\mathrm{O}-\mathrm{H}$ bond. The gradient values typical for the states above the DIP are nearly twice as large as the gradients of the dissociative states below the DIP. This indicates the presence of even faster nuclear dynamics in such multiply-excited states.

In our simulations considering a mixture of discrete and continuum states above the DIP, we assumed a state discrete, if at least $75 \%$ of a CI state correspond to multiple-excitation configurations. Otherwise, the state is considered as continuum. Figure 2(b) shows the results of our simulations for two values of FC broadening applied to the region above the DIP. With a relatively low broadening value of $0.4 \mathrm{eV}$ FWHM (solid green line), a clear peak is revealed at the binding energy of $1187 \mathrm{eV}$ in close agreement with the position of the most intense experimental peak. Furthermore, the simulations demonstrate the presence of structures around $1180 \mathrm{eV}$ and in the $1190-1200 \mathrm{eV}$ region, which is also in line with the experimental observation.

Applying a broadening value of $4 \mathrm{eV} \mathrm{FWHM}$ to the region above the DIP, consistent with the theoretical FC widths indicated in table $\mathbb{1}$, leads to smoother features in the simulated spectrum (dotted red line) and reproduces fairly well the experimental spectral shape in this region.

One can assume that the agreement could be further improved considering the coupling between the discrete and continuum states above the DIP, where the Fano interference may lead to modulation of the shape and relative intensities of the spectral features. However, this treatment is beyond the scope of the present paper.

\section{CONCLUSION}

In conclusion, in this work we present a combined experimental and theoretical study of the DCH photoelectron spectrum in gas-phase water molecules ionized with hard x-rays above the oxygen $K^{-2}$ DIP. We present here a detailed analysis of both the below- and the above-DIP regions. At the binding energies below the DIP, the spectrum contains features that we assign to pre-edge $K^{-2} V$ states. High-resolution measurements allowed for a detailed comparison of the spectral line shapes with theoretical calculations, which reveals dissociative nuclear dynamics in the pre-edge $K^{-2} V$ states. To interpret the spectral features above the DIP, the nature (continuum or discrete) of the numerous states contributing to this 
region has to be taken into account, together with a strong configuration-interaction mixing between a high number of discrete states. Theoretical results have been obtained which detail the extent of such mixing. The experimental photoelectron spectrum exhibits broad spectral lines at the binding energies above the $K^{-2}$ DIP, which are attributed to highly excited DCH states, involving multiple shake-up excitation processes. A strong broadening caused by the nuclear motion indicates a highly dissociative nature of these multiply excited states in agreement with the theoretical analysis.

\section{ACKNOWLEDGMENTS}

Experiments were performed on the GALAXIES beamline at SOLEIL Synchrotron, France (Proposal No. 20150921, 99150083). We are grateful to SOLEIL staff for smoothly running the facility. T.M. and J.M. acknowledge financial support from the French Agence Nationale de la Recherche (ANR) through the ATTOMEMUCHO project (ANR-16-CE300001).

[1] J. H. D. Eland, M. Tashiro, P. Linusson, M. Ehara, K. Ueda, and R. Feifel, Phys. Rev. Lett. 105, 213005 (2010).

[2] P. Lablanquie, F. Penent, J. Palaudoux, L. Andric, P. Selles, S. Carniato, K. Bučar, M. Žitnik, M. Huttula, J. H. D. Eland et al., Phys. Rev. Lett. 106, 063003 (2011).

[3] P. Lablanquie, T. P. Grozdanov, M. Žitnik, S. Carniato, P. Selles, L. Andric, J. Palaudoux, F. Penent, H. Iwayama, E. Shigemasa et al., Phys. Rev. Lett. 107, 193004 (2011).

[4] P. Linusson, O. Takahashi, K. Ueda, J. H. D. Eland, and R. Feifel, Phys. Rev. A 83, 022506 (2011).

[5] M. Mucke, J. H. D. Eland, O. Takahashi, P. Linusson, D. Lebrun, K. Ueda and R. Feifel, Chem. Phys. Lett. 558, 82 (2013).

[6] M. Nakano, P. Selles, P. Lablanquie, Y. Hikosaka, F. Penent, E. Shigemasa, K. Ito, and S. Carniato, Phys. Rev. Lett. 111, 123001 (2013).

[7] S. Carniato, P. Selles, L. Andric, J. Palaudoux, F.Penent, M. Žitnik, K. Bučar, M. Nakano, 
Y. Hikosaka, K. Ito et al., J. Chem. Phys. 142, 014307 (2015).

[8] S. Carniato, P. Selles, L. Andric, J. Palaudoux, F.Penent, M. Žitnik, K. Bučar, M. Nakano, Y. Hikosaka, K. Ito et al., J. Chem. Phys. 142, 014308 (2015).

[9] N. Berrah, L. Fang, B. Murphy, T. Osipov, K. Ueda, E. Kukk, R. Feifel, P. van der Meulen, P. Salen, H. T. Schmidt, R. D. Thomas, M. Larsson, R. Richter, K. C. Prince, J. D. Bozek, C. Bostedt, S.Wada, M. N. Piancastelli, M. Tashiro, and M. Ehara, Proc. Natl. Acad. Sci. U. S. A. 108, 16912 (2011).

[10] P. Salén, P. van der Meulen, H. T. Schmidt, R. D. Thomas, M. Larsson, R. Feifel, M. N. Piancastelli, L. Fang, B. Murphy, T. Osipov et al., Phys. Rev. Lett. 108,199903 (2012).

[11] M. Larsson, P. Salén, P. vd Meulen, H. T. Schmidt, R. D. Thomas, R. Feifel, M. N. Piancastelli, L. Fang, B. Murphy, T. Osipovet al., J.Phys.B: At. Mol. Opt. Phys. 46, 164030 (2013).

[12] V. Zhaunerchyk, M. Mucke, P. Salén, P. vd Meulen, M. Kaminska, R. J. Squibb, L. J. Frasinski, M. Siano, J. H. D. Eland, P. Linusson et al., J.Phys.B: At. Mol. Opt. Phys. 46, 164034 (2013).

[13] L.J.Frasinski, V. Zhaunerchyk, M. Mucke, R. J. Squibb, M. Siano, J. H. D. Eland, P. Linusson, P. vd Meulen, P. Salén, R. D. Thomas et al., Phys. Rev. Lett. 111, 073002 (2013).

[14] L. S. Cederbaum, F. Tarantelli, A. Sgamellotti, and J. Schirmer, J. Chem. Phys. 85, 6513 (1986).

[15] R. Püttner, G. Goldsztejn, D. Céolin, J.-P. Rueff, T. Moreno, R. Kushawaha, T. Marchenko, R. Guillemin, L. Journel, D. W. Lindle et al., Phys. Rev. Lett. 114, 093001 (2015).

[16] G. Goldsztejn, T. Marchenko, R. Püttner, L. Journel, R. Guillemin, S. Carniato, P. Selles, O. Travnikova, D. Céolin, A. F. Lago et al., Phys. Rev. Lett. 117, 133001 (2016).

[17] G. Goldsztejn, R. Püttner, L. Journel, R. Guillemin, O. Travnikova, B. Cunha de Miranda, I. Ismail, S. Carniato, P. Selles, D. Céolin et al., Phys. Rev. A 96, 012513 (2017).

[18] R. Feifel, J. H. D. Eland, S. Carniato, P. Selles, R. Püttner, D. Koulentianos, T. Marchenko, L. Journel, R. Guillemin, G. Goldsztejn et al., Sci. Rep. 7, 13317 (2017)

[19] D. Koulentianos, R. Püttner, G. Goldsztejn, T. Marchenko, O. Travnikova, L. Journel, R. Guillemin, D. Céolin, M. N. Piancastelli, M. Simon et al., Phys. Chem. Chem. Phys. 20, 2724 (2018)

[20] S. Carniato, P. Selles, P. Lablanquie, J. Palaudoux, L. Andric, M. Nakano, Y. Hikosaka, K. Ito, T. Marchenko, O. Travnikova et al., Phys. Rev. A 94, 013416 (2016).

[21] D. Koulentianos, S. Carniato, R. Püttner, G. Goldsztejn, T. Marchenko, O. Travnikova, L. 
Journel, R. Guillemin, D. Céolin, M. L. M. Rocco et al., J.Chem.Phys. 149, 134313 (2018).

[22] T. Marchenko, G. Goldsztejn, K. Jänkälä, O. Travnikova, L. Journel, R. Guillemin, N. Sisourat, D. Céolin, M. Žitnik, M. Kavčič et al., Phys. Rev. Lett. 119, 133001 (2017).

[23] O. Travnikova, T. Marchenko, G. Goldsztejn, K. Jänkälä, N. Sisourat, S. Carniato, R. Guillemin, L. Journel, D. Céolin, R. Püttner et al., Phys. Rev. Lett., 116, 213001 (2016).

[24] O. Travnikova, N. Sisourat, T. Marchenko, G. Goldsztejn, R. Guillemin, L. Journel, D. Céolin, I. Ismail, A. Lago, R. Püttner et al., Phys. Rev. Lett. 118, 213001 (2017).

[25] E.F.Garman and M.Weik, J. Synchrotron Rad. 26, 907 (2019)

[26] T. Marchenko, L. Inhester, G. Goldsztejn, O. Travnikova, L. Journel, R. Guillemin, I. Ismail, D. Koulentianos, D. Céolin, R. Püttner et al., Phys. Rev. A 98, 063403 (2018).

[27] D. Céolin, J. M. Ablett, D. Prieur, T. Moreno, J.-P. Rueff, T. Marchenko, L. Journel, R. Guillemin, B. Pilette, T. Marin et al., J. Electron Spectrosc. Relat. Phenom. 190, 188 (2013).

[28] J.-P. Rueff, J. M. Ablett, D. Céolin, D. Prieur, T. Moreno, V. Balédent, B. Lassalle, J. E. Rault, M. Simon, and A. Shukla, J. Synchrotron Rad. 22, 175 (2015).

[29] W. E. Moddeman, T. A. Carlson, M. O. Krause, B. P. Pullen, W. E. Bull and G. K. Schweitzer, J. Chem. Phys. 55, 2317 (1971).

[30] L. Inhester, C. F. Burmeister, G. Groenhof, and H. Grubmüller, J. Chem. Phys. 1365, 144304 (2012).

[31] M. W. Schmidt et al., J. Comput. Chem. 14, 1347 (1993).

[32] H. Ågren, V. Caravetta, and L. G. M Pettersson, Theor. Chem. Acc. 97, 14 (1997).

[33] S. Carniato, J. of Electron Spec. and Relat. Phenom., 339, 146931 (2020).

[34] I. Hjelte, M. N. Piancastelli, R. F. Fink, O. Björneholm, M. Bässler, R. Feifel, A. Giertz, H. Wang, K. Wiesner, A. Ausmees et al., Chem. Phys. Lett. 334, 151 (2001).

[35] R. Püttner, T. Arion, M. Förstel, T. Lischke, M. Mucke, V. Sekushin, G. Kaindl, A. M. Bradshaw, and U. Hergenhahn, Phys. Rev. A 83, 043404 (2011). 